\title{
DNA IMAGE CYTOMETRY IN PROGNOSTICATION OF COLORECTAL CANCER: PRACTICAL CONSIDERATIONS OF THE TECHNIQUE AND INTERPRETATION OF THE HISTOGRAMS
}

\author{
ABDElbaset BuHMEIDA ${ }^{1}$, YRJÖ COLLAN ${ }^{2}$, KARI SYRJÄNEN ${ }^{1}$ AND SEPPO PYRHÖNEN ${ }^{1}$ \\ ${ }^{1}$ Department of Oncology and Radiotherapy; ${ }^{2}$ Department of Pathology, Turku University Central Hospital, \\ Turku, Finland \\ e-mail: abuhme@utu.fi \\ (Accepted October 21, 2005)
}

\begin{abstract}
The role of DNA content as a prognostic factor in colorectal cancer (CRC) is highly controversial. Some of these controversies are due to purely technical reasons, e.g. variable practices in interpreting the DNA histograms, which is problematic particularly in advanced cases. In this report, we give a detailed account on various options how these histograms could be optimally interpreted, with the idea of establishing the potential value of DNA image cytometry in prognosis and in selection of proper treatment. Material consists of nuclei isolated from $50 \mu \mathrm{m}$ paraffin sections from 160 patients with stage II, III or IV CRC diagnosed, treated and followed-up in our clinic. The nuclei were stained with the Feulgen stain. Nuclear DNA was measured using computer-assisted image cytometry. We applied 4 different approaches to analyse the DNA histograms: 1) appearance of the histogram (ABCDE approach), 2) range of DNA values, 3) peak evaluation, and 4) events present at high DNA values. Intra-observer reproducibility of these four histogram interpretation was $89 \%, 95 \%, 96 \%$, and $100 \%$, respectively. We depicted selected histograms to illustrate the four analytical approaches in cases with different stages of $\mathrm{CRC}$, with variable disease outcome. In our analysis, the range of DNA values was the best prognosticator, i.e., the tumours with the widest histograms had the most ominous prognosis. These data implicate that DNA cytometry based on isolated nuclei is valuable in predicting the prognosis of CRC. Different interpretation techniques differed in their reproducibility, but the method showing the best prognostic value also had high reproducibility in our analysis.
\end{abstract}

Keywords: colorectal cancer, DNA cytometry, histogram, interpretation, predictive value, prognosis, technical notes.

\section{INTRODUCTION}

Several clinical and pathological variables are useful in assessing the prognosis of colorectal cancer (CRC) patients, but the prediction is far from perfect. Therefore, an active search is ongoing for new prognostic and predictive tools capable of identifying high-risk patients who would benefit from individually tailored treatment options (Galizia et al., 2002; Bendardaf et al., 2004a). Lately, there has been an increasing interest in DNA quantification (Bazan et al., 2002).

Indeed, abnormalities in DNA ploidy are seen in many human tumors, and determination of ploidy and proliferative activity has been shown to provide valuable prognostic information (Cohen, 1996; Millot and Dufer, 2000). Until now, however, the prognostic value of DNA image ploidy has not been fully explored in CRC patients (Salud et al., 1999), partly because of substantial nuclear overlaps in the tumour sections.
At present, the role of DNA content as a prognostic factor in CRC is highly controversial (Silvestrini, 2000). While several studies have suggested that DNA ploidy is an independent prognostic factor (Scott et al., 1987; Witzig et al., 1991; Lanza et al., 1998), others have reported that DNA content is not associated with clinical outcome (Zarbo et al., 1997, Tonouchi et al., 1998). Part of these discrepant observations might be explained by the inconsistencies and true differences in the technical aspects of recording the DNA contents. This is clearly shown by our previous study, where only some of the analytical approaches used to assess the DNA histograms resulted in prognostically valuable information (Buhmeida et al., submitted). It is also well recognised that CRCs consist of many different subpopulations of tumor cells with different DNA content (Petersen et al., 1979; Tribukait et al., 1983)

A feasible explanation for these controversies might be the variations in the genomic instability of 
CRC. Our previous study suggested that this variation was typically associated with wide histograms. Indeed, DNA values over a wide range are associated with the ability of tumour cells to survive, grow, and metastasize (Geido et al., 2002). DNA aneuploidy as such is a known biological marker of the oncogenic potential of colorectal adenomas (CRA). Accumulation of genetic alterations of cancer-related genes is also essential in colorectal carcinogenesis (Fearon and Vogelstein, 1990; Aaltonen, 1996; Tomlinson et al., 1997). However, it is unclear whether there is any relationship between these genetic alterations in progression of CRAs and early CRCs (Sugai et al., 2003). Only a small number of studies have analysed genomic instability in relation to DNA histogram features of CRC (Hawkins et al., 2001, Giaretti et al., 2003)

Interpretation of the histograms from advanced cancers is problematic, however. In this study, we give a detailed presentation on how these histograms can and should be interpreted in a consistent manner. This is particularly important in the prospective studies evaluating the importance of DNA image cytometry in CRC.

\section{PATIENTS AND METHODS}

The present patient material comprises a series of 160 patients diagnosed and treated for Stage II, Stage III, and Stage IV (Dukes B, C, and D) colorectal cancer (CRC) at the Department of Oncology and Radiotherapy, Turku University Central Hospital and six other hospitals in the same hospital district, between January 1996-December 1997. The numbers of patients were as follows: stage II 95, stage III 28, and stage IV 37. The patients were seen at 6 month intervals until death or conclusion of the follow-up time. The mean follow-up time for the whole series was 53.9 (SD 24.8) months (median 61.3 month).

\section{FEULGEN STAINING}

One hundred and sixty formalin-fixed, paraffin embedded cancer samples were obtained. Sections were cut serially at a thickness of $5 \mu \mathrm{m}$ for routine haematoxylin and eosin staining. Based on the original pathology reports, conventional histological slides were examined from blocks which appeared to contain a satisfactory proportion $(60 \%)$ of tumour cells. Only samples with predominance of cancer tissue were accepted for the study. Normal stromal diploid cells were invariably present in the samples, but they represented a minority. For the Feulgen staining, nuclei were isolated from 50 - $\mu \mathrm{m}$-thick paraffin sections according to the method described by Hedley et al (1985), in which the sections are placed in glass tubes and dewaxed in xylene, following which they are rehydrated by sequential immersion in $100 \%, 95 \%$, $70 \%$, and $50 \%$ ethanol, washed in water, and treated with $0.5 \%$ pepsin (activity 2,500-3,200 units per mg protein), adjusted to $\mathrm{pH} 1.5$, in a $37^{\circ} \mathrm{C}$ water bath. The isolated nuclei were cytocentrifuged on glass slides and stained with Feulgen stain according to the method of Gaub et al. (1975). The samples were washed in distilled water, followed by acid hydrolysis in $5 \mathrm{M}$ hydrochloric acid at room temperature $\left(20^{\circ} \mathrm{C}\right)$ for one hour. After washing in distilled water, samples were treated in darkness with Schiff's reagent (stain: pararosaniline) for $2 \mathrm{hrs} 45 \mathrm{~min}$ at room temperature $\left(20^{\circ} \mathrm{C}\right)$, rinsed in distilled water, treated for $3 \times 10$ minutes in fresh aqueous sodium tiosulphate $(180 \mathrm{ml}$ distilled water, $10 \mathrm{ml} 1 \mathrm{M} \mathrm{HCl}, 10 \mathrm{ml} 10 \% \mathrm{Na}_{2} \mathrm{~S}_{2} \mathrm{O}_{5}$ ), and rinsed for $5 \mathrm{~min}$. The Feulgen reaction labelled DNA magenta. The intensity of the stain in these samples is proportional to the amount of DNA present. After dehydration the smears were treated with xylene and mounted, then stored in darkness.

\section{IMAGE ANALYSIS CYTOMETRY}

The intensity of Feulgen staining was measured using a computer-assisted image analysis cytometry system with a Nikon microscope (Eclipse E 400; Japan) utilizing AHRENS ICM, which is a cytometry system based on Microsoft Windows for analysis of DNA content or immunostaining (designed and produced by Olaf Ahrens; Meßtechnische Beratung, Bargteheide/Hamburg, Germany). The field of view from the camera (JAI DSP surveillance color CCD camera, CV-S 3200/3300) was stored in image memory with resolution of 736 by 560 pixels. The image was produced by a plan objective (Nikon; X 40) and the measurements were made from that image. Prior to each measurement session the illumination of the microscope was adjusted according to the method of Köhler (Bohnhoff, 1979). Several histograms were produced twice, and the histograms turned out to be very similar.

\section{INTERPRETATION OF THE DNA HISTOGRAMS}

Feulgen-stained samples were available from the primary tumours of all 160 patients. The whole slide was scanned at $10 \times$ objective to find the most suitable area or areas for analysis, with atypical cells, when possible. The selected area or areas were scanned at 
$40 \times$ objective, and an average of 10-15 microscopic fields were selected, and at most 200 consecutive tumour nuclei with clear nuclear borders were outlined and measured. Overlapping or cut (from surfaces of the $50 \mu \mathrm{m}$ section) nuclei were not measured. In addition, 30 small lymphocytes were measured as internal controls. The internal (lymphocytes) control peaks represent the location of the $2 \mathrm{c}$ diploid peak ( $\mathrm{c}=$ haploid DNA content). The control peak was always narrow with small variation (coefficient of variation $<5 \%$ ).

We applied four different approaches for analysis of the histograms obtained in DNA cytometry, as follows:

1. ABCDE approach (The general appearance of the histogram)

This approach classified the histograms according to the location and appearance of the peaks into different groups (A, B, C, D, and E) (Bendardaf et al., 2004b).

A: The histogram showed one dominant diploid or peridiploid peak (mode within 1.7c-2.3c). No other prominent peaks were present.

B: The histograms with a dominant tetraploid or peritetraploid peak (mode within 3.41c-4.41c), or a broad peak with the mode centering on $4 \mathrm{c}$.

$\mathrm{C}$ : The histograms with prominent peak between the $2 \mathrm{c}$ and $4 \mathrm{c}$ regions (mode within $2.31 \mathrm{c}-3.4 \mathrm{c}$ ).

D: The histogram had a narrow or broad peak with mode above $4.41 \mathrm{c}$, or there were several prominent peaks.

E: The other histograms not belonging to any of the above categories. Histograms with too few cells to show any peaks.

2. Range evaluation approach (The width of the histogram)

In this approach, we concentrated mainly on the range of the histograms, i.e., the width of the histograms. We classified the histograms as being distributed within 12 different gates as follows:
$1.8 \mathrm{c}-4.4 \mathrm{c}$
$3.0 \mathrm{c}-4.4 \mathrm{c}$
$1.8 \mathrm{c}-5.0 \mathrm{c}$
$3.0 \mathrm{c}-5.0 \mathrm{c}$
$1.8 \mathrm{c}-7.0 \mathrm{c}$
$3.0 \mathrm{c}-7.0 \mathrm{c}$
$1.8 \mathrm{c}-9.0 \mathrm{c}$
$3.0 \mathrm{c}-9.0 \mathrm{c}$
$1.8 \mathrm{c}-10.0 \mathrm{c}$
$3.0 \mathrm{c}-10.0 \mathrm{c}$
$1.8 \mathrm{c}->10 \mathrm{c}$
$3.0 \mathrm{c}->10 \mathrm{c}$

The first six were also grouped together as lowstart gates ( $1.8 \mathrm{c}$ upwards) ( $=1 \mathrm{st}$ set), and the last 6 as high-start gates (3.0c upwards) $(=2$ nd set). The absolute width of the histogram was also measured. On this basis, the gates were divided into 2 groups: A (width $1.4-6.0$ units, narrow range) consisting of gates $1.8 \mathrm{c}-4.4 \mathrm{c}, 1.8 \mathrm{c}-5.0 \mathrm{c}, 1.8 \mathrm{c}-$ $7.0 \mathrm{c}, 3.0 \mathrm{c}-4.4 \mathrm{c}, 3.0 \mathrm{c}-5.0 \mathrm{c}, 3.0 \mathrm{c}-7.0 \mathrm{c}, 3.0 \mathrm{c}-$ 9.0c; and $\mathrm{B}$ (width 6.1->10 units, wide range) including the gates $1.8 \mathrm{c}-9.0 \mathrm{c}, 1.8 \mathrm{c}-10.0 \mathrm{c}, 1.8 \mathrm{c}-$ $>10 \mathrm{c}, 3.0 \mathrm{c}-10.0 \mathrm{c}, 3.0 \mathrm{c}->10 \mathrm{c}$. This classification is called the 2-tier system. In addition, a 3-tier grading was tested classifying the width of the histograms into narrow (1.4-3.0 units), intermediate (3.1-6.0 units) and broad (6.1->10 units) range categories, following the same principles.

3. Peak evaluation (Peaks grouped according to mode of the peak)

In this approach, we classified the samples into different categories according to the location of the mode of the peak(s) in the histogram. The gates describing the location were:

$$
\begin{aligned}
& 1.7 c-2.3 c \\
& 2.3 c-3.7 c \\
& 3.7 c-4-3 c \\
& 4.3 c-6.0 c \\
& 6.0 c-7.7 c
\end{aligned}
$$

\section{Events at high DNA values}

In this approach we calculated the fraction of the cells above certain cut-off points. We selected the following cut-offs for the analysis:

$$
\begin{aligned}
& >5 \mathrm{c} \\
& >6 \mathrm{c} \\
& >7 \mathrm{c} \\
& >8 \mathrm{c} \\
& >9 \mathrm{c}
\end{aligned}
$$

\section{REPRODUCIBILITY OF THE METHOD}

The histograms were evaluated by the first author by using the 4 approaches at one time point. After a period of 4 weeks, the evaluation of the same histograms was blindly repeated again. Reproducibility of the evaluation of the histograms was $89 \%$ for the $A B C D E$ approach, $95 \%$ for the range evaluation approach, $96 \%$ for peak's evaluation approach, and $100 \%$ for the events at high DNA values approach. 


\section{EXAMPLES OF INTERPRETATION OF THE HISTOGRAMS}

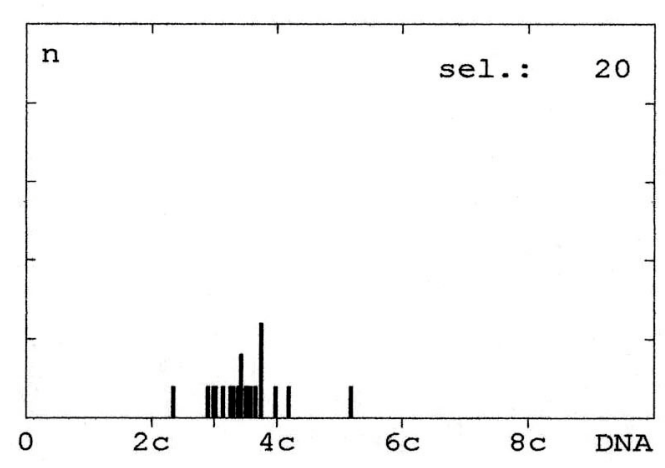

A

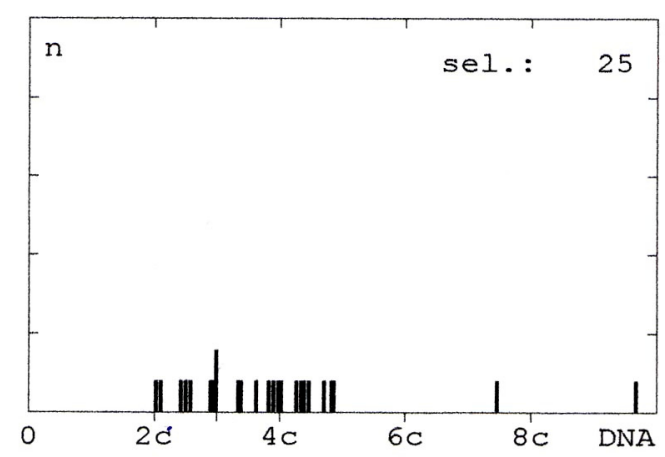

B

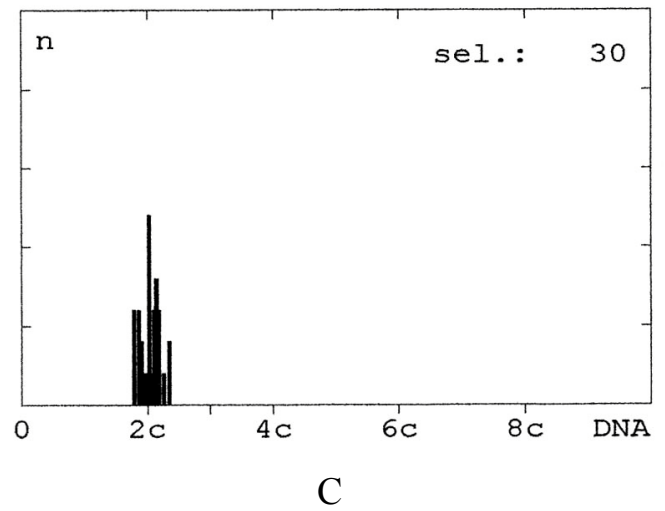

Fig. 1. Histograms with limited number of cells. (A) In this histogram, the cells were too few for histogram peak to emerge. DNA values are distributed mainly at the peri-tetraploid region. According to ABCDE approach, this histogram belongs to group $E$ (the cells were too few for histogram peaks to emerge). The patient was a 71-year-old male with stage IV, Ggrade $3 \mathrm{CRC}$, and he died of the disease 5 months after diagnosis. This histogram belongs among the narrow range histograms. (B) Cells were too few for the histogram peak to emerge. The range of DNA values falls between 1.8c-10c. The patient was a 72-year-old male with stage II, Grade 2 adenocarcinoma. He is alive and free of disease after 73 months from the diagnosis. (C) Diploid histogram. There are no values outside the diploid range. Even though the number of cells studied is low, this type of histogram without any evidence of non-diploidy can be considered diploid. The patient, an 82-year-old woman had stage III Grade 2 adenocarcinoma of the colon, and she is still alive, disease-free, 72 months after the original diagnosis. By definition, this histogram belongs among the narrow range histograms.

Fig. 1. shows histograms which could not be evaluated in all approaches (Figs. 1A,B). The reason was that enough nuclei could not be found for the analysis. If DNA values were distributed over a wider range, but were so few that no peaks were detected, evaluation was considered uncertain. However, because $>5 \mathrm{c}$ cells could be present, such samples could suggest a category in the "events at high DNA values"approach. Fig. 1C shows that there are cases in which a pure diploid peak can appear clearly after a limited number of studied nuclei. Fig. 1C can be interpreted to present a diploid nuclear population, but because of the limited number of nuclei, the decision may be uncertain. In this material, samples with diploid peak only were extremely rare (4/160).

Fig. 2 shows cases with dominant tetraploid peaks. Such peaks could be associated with a proliferative cell population at $8 \mathrm{c}$ (Figs. 2C,D). The latter types of histograms were clearly wider than histograms with the tetraploid peak only. In our series, there were $45 / 160$ cases with a dominant tetraploid peak or dominant tetraploid peak plus a proliferative peak.

In addition to dominant diploid and tetraploid peaks, there were a substantial number of cases $(71 / 160)$ with dominant aneuploid peaks (Fig. 3). These were usually associated with corresponding proliferative cell population. 


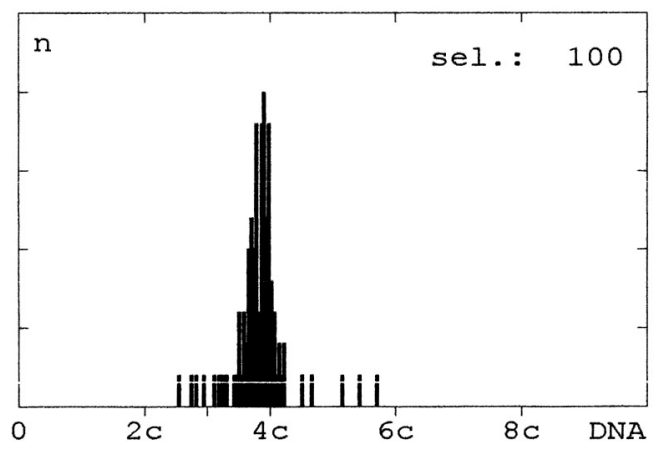

$1 \mathrm{~A}$

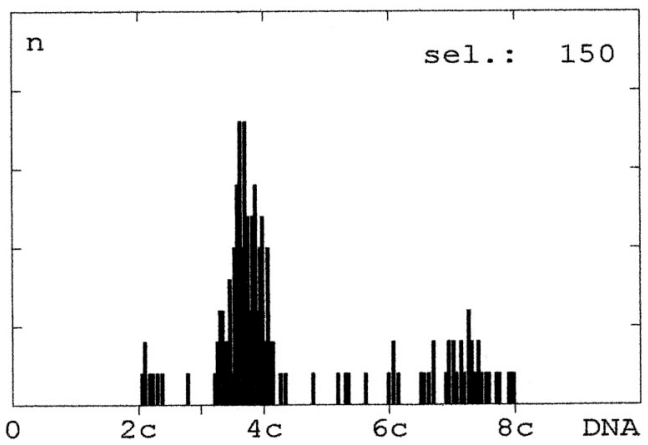

$1 \mathrm{C}$

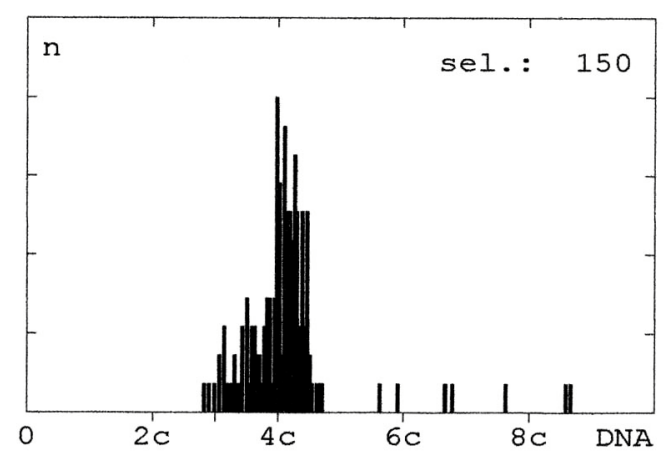

1B

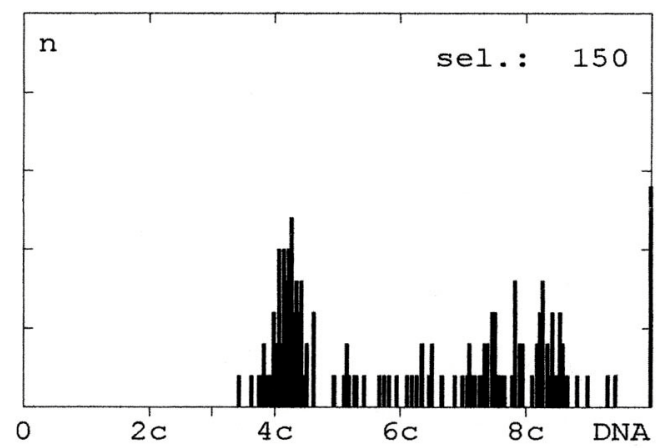

1D

Fig. 2. Histograms showing tetraploid or peri-tetraploid peaks. (A) Dominant tetraploid/peri-tetraploid peak, only a few nuclei outside the peri-tetraploid region (3.4c-4.4c). There are no diploid nuclei. Lower than tetraploid/peri-tetraploid values and higher than tetraploid/peri-tetraploid values may be due to unevenly separating chromosomes in mitosis (Steinbeck, 1998) or proliferation. Around 3\% of 100 nuclei fall into the category of $>5 \mathrm{c}$ nuclei. The patient, a 68-year-old man had stage II, Grade 1 CRC, and he died of unrelated cause 44 months after diagnosis. According to the 2-tier system, this histogram belongs among the narrow range histograms. (B) Tetraploid/peri-tetraploid peak with numerous cells outside the peri-tetraploid region. In addition, numerous nuclei show values above $5 c(10 \%), 6 c(7 \%)$, and $8 c(3 \%)$. Values between $2 c$ and $4 c$ can be expected to present nuclei after uneven mitotic divisions or proliferation. The patient was a 56-year-old woman with stage II, Grade 2 carcinoma, and who is alive without disease after 68 months from the original diagnosis. This histogram belongs to group $B$ when evaluated with the ABCDE approach, and represents another wide range histogram. (C) Prominent tetraploid/peri-tetraploid peak slightly shifted towards diploidy. There are also two other prominent peaks (near diploid and $7.5 \mathrm{c}$ regions) placing this in category $D$ in the $A B C D E$ evaluation. Diploid cells are few and obviously do not present a feeding population to tetraploid/peritetraploid cells. On the other hand, the flat peak at $7.5 \mathrm{c}$ clearly is reflecting the proliferating cells of the tetraploid/peri-tetraploid population. The range of this histogram falls into the category of $1.8 c-9 c$. The patient was a 72-year-old woman, with stage IV, Grade 2 adenocarcinoma, and she died of the disease at 27 months. Another example of a wide range histogram. (D) Tetraploid/peri-tetraploid peak slightly shifted towards higher than $4 c$ cells. The values above $5 c$ are numerous ( $>5 c 50 \%,>6 c 40 \%,>7 c 30 \%,>8 c 20 \%$, and $>9 c 10 \%)$. The dominant population of cells is probably slightly higher than tetraploid in their DNA content. The flat peak around 8c may reflect proliferative cells of the dominant population. The patient was a 58-year-old woman, with stage II, Grade 2 CRC, who is alive and disease-free, 68 months after the original diagnosis. Also a wide range histogram. 


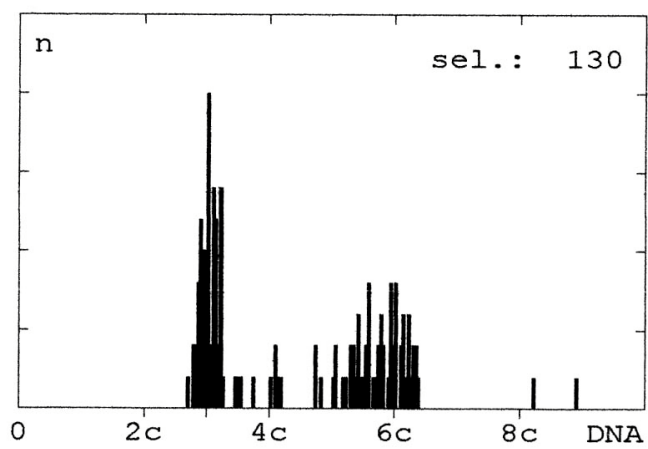

$1 \mathrm{~A}$

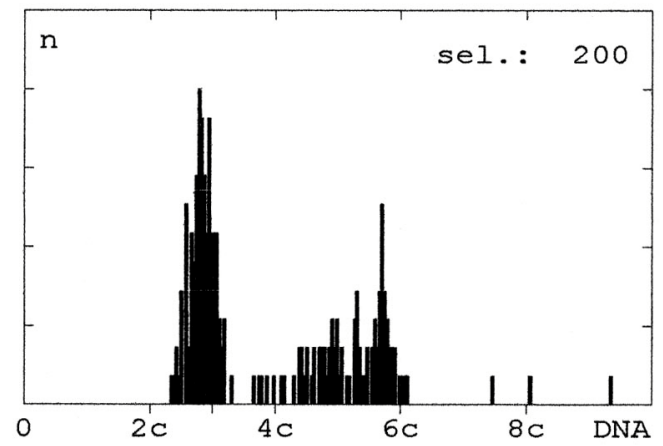

$1 \mathrm{C}$

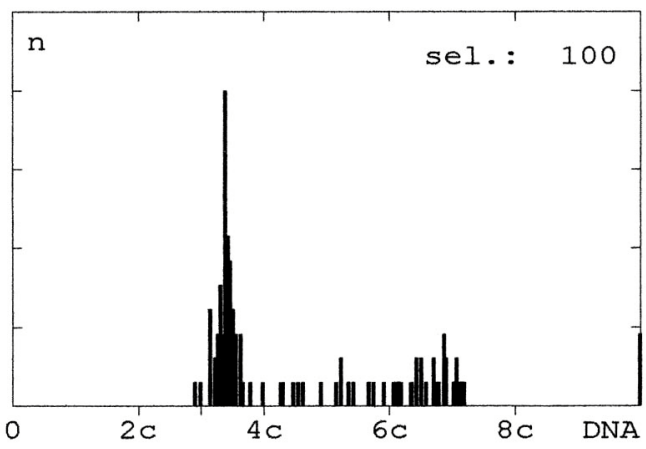

$1 \mathrm{~B}$

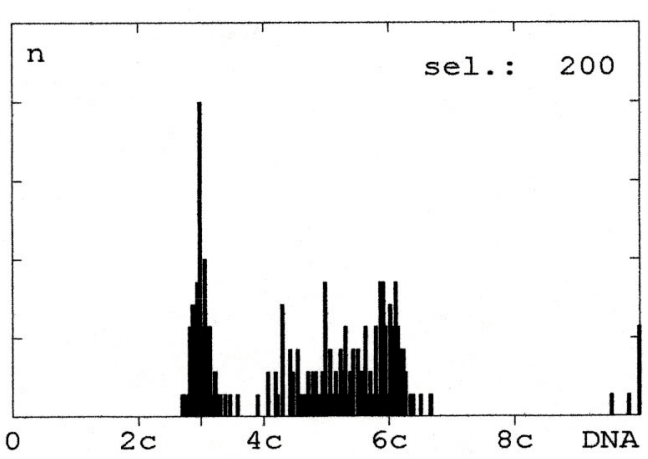

$1 \mathrm{D}$

Fig. 3. Histograms of aneuploid peaks with corresponding proliferative peaks. (A) Prominent peak at 3c region with a broad peak at $6 c$ that may reflect the proliferative cells of a dominant population. The histogram characteristics are those of group C. A few nuclei are located at the tetraploid region. The patient is a 35-yearold woman with stage III CRC (Grade 2), who is alive and well after 59 months. This histogram belongs to wide range histograms. (B) Prominent aneuploid peak at $3.5 \mathrm{c}$ with small peak at $7 c$, which may reflect proliferative dominant cells. The nuclei distributed between $4 c$ and $6 c$ may be due to uneven mitotic divisions, or reflect proliferation. The histogram would be interpreted as group $C$ in the ABCDE approach. The range of the DNA values falls between $3 c-10 c$ (note 2 nuclei at 10c). The patient, 86-year-old man had stage II, Grade 2 disease and he died of other cause 41 months after the diagnosis. Also this histogram belongs to the wide range histogram category. (C) Prominent peak below $3 c$ with another peak below $6 c$, which most likely reflects the proliferation of the dominant cell population. There is also small broad peak around $5 c$ region. The range of DNA values is between $1.8 \mathrm{c}-10 \mathrm{c}$ with $30 \%$ of cells above $5 \mathrm{c}$. According to the ABCDE system, this histogram belongs to group D. The patient was a 59-year-old woman with stage II (Grade 1) disease, who died of disease 15 months after diagnosis. Another example of a wide range histogram. (D) Prominent peak at $3 c$ with broad peaks at $5 \mathrm{c}$ and $6 \mathrm{c}$ regions. The peak at $6 \mathrm{c}$ may reflect the proliferating cells of the dominant cell populations at $3 c$, and the broad peak between $4 c-5 c$ could denote uneven mitotic divisions. Proliferating cells are also seen at 10c. The histogram belongs to group D with 50\% of the cells above $5 \mathrm{c}$. The range of the histogram falls between 1.8c-10c. This patient is a 76-year-old female, with stage II, Grade 2 adenocarcinoma, and she is alive, disease-free after 71 months from the diagnosis. Also this histogram belongs among the category of wide range histograms. 


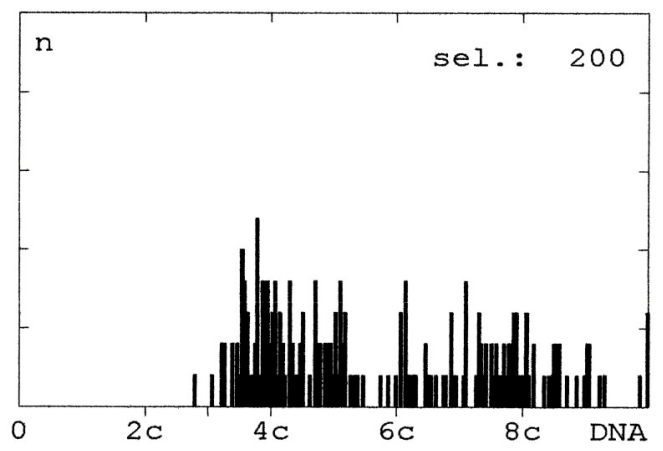

$1 \mathrm{~A}$

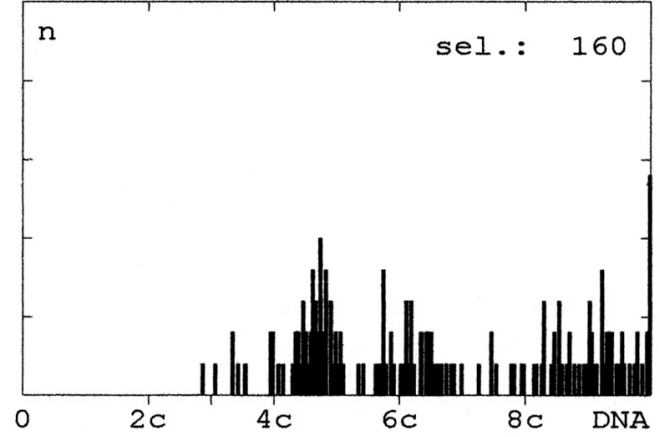

$1 \mathrm{~B}$

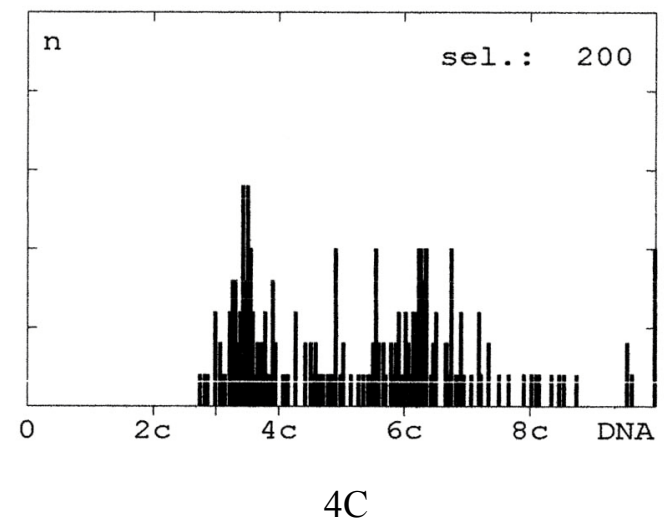

Fig. 4. Histograms of "New York Skyline"' pattern. (A) Multiple broad aneuploid peaks of numerous DNA values are seen over the whole range of the histogram. The histogram represents group $D$ in the ABCDE system, with a range of 3c-10c. The patient was a 49-year-old female with stage II, Grade 2 CRC, who died of the disease in 10 months. Another wide range histogram. (B) Multiple broad aneuploid peaks of numerous $D N A$ values are seen over the whole range of the histogram. The histogram belongs to group $D$ according to $A B C D E$ approach with range of the histogram between $3 c->10 c$. The patient is a 43-year-old male with stage II (Grade 2) disease, and he was alive and well at 89 months after diagnosis. A typical example of the wide range histograms. (C) Multiple broad aneuploid peaks of numerous DNA values are seen over the whole range of the histogram. This group D histogram has a range between 3c->10c. The patient was an 85-year-old female with stage II adenocarcinoma of the rectum, who died of the disease 19 months after the diagnosis. Wide range histogram like above.

The cancers with typical histogram showing peaks (diploid or peri-diploid, tetraploid or peri-tetraploid, and aneuploid peaks) - after progression - seem to merge into histograms with the " New York skyline", appearance. Often in the latter you can imagine a tetraploid or an aneuploid peak, but the continuity of events over the scale, without practically any interruption between events defined such cases as belonging to the "NY skyline" category (group D, Fig. 4). We had $(18 / 160)$ cases with this histogram profile in our material.

\section{STATISTICAL ANALYSIS}

Statistical analyses were performed using the SPSS $^{\circledR}$ (SPSS, Inc., Chicago, USA) and STATA (Stata
Corp., Texas, USA) software packages (SPSS for Windows, version 12.0.1 and STATA/SE 9.1). Frequency tables were analysed using the Chi-square test, with likelihood ratio (LR) or Fischer's exact test to assess the correlation between the categorical variables. Differences in the means of continuous variables were analysed using non-parametric tests (Mann-Whitney) or ANOVA (analysis of variance). Logistic regression models were used to analyse the power of different covariates as predictors of the outcome variables, calculating crude ORs (and 95\% CI) in univariate analysis. Univariate survival (life-table) analysis for the outcome measure (DSS, DFS) was based on Kaplan-Meier method. Survival analysis was also run by using Cox's proportional hazards model (both in univariate mode for continuous variables and 
in multivariate mode) in a backward stepwise manner with the log-likelihood ratio (L-R) significance test, and using the default values for enter and exclusion criteria. The assumption of proportional hazards was controlled by log-minus-log (LML) survival plots. In all tests, the values $p<0.05$ were regarded statistically significant.

\section{RESULTS}

$A B C D E$ approach. In our series, the $\mathrm{ABCDE}$ approach proved to be of no statistical significance in predicting patient survival $(\mathrm{p}=0.728)$.

Range evaluation approach. The low start gates and the high start gates were significantly different in respect to disease outcome. When all cases were analysed in terms of the 5-year disease-free survival (DFS), the association was significant $(\mathrm{p}<0.010)$.

When the follow-up time covered the whole observation period, the results lost their significance $(\mathrm{p}<0.108)$, because the survival curves overlapped after 6 years (Fig. 5). At best (at 4-5 years), the difference in survival was almost $25 \%$. Diseasespecific survival (DSS) did not reach statistical significance in the 2 -tier system $(\mathrm{p}<0.08)$. The 3 -tier system showed that narrow and intermediate widths very much carried the same prognostic information suggesting that the 2-tier system should be applied in clinical practice.

Because stage IV disease with distant metastasis has per se worse prognosis than stage II and III disease, the former were excluded from the analysis. In this series with stage II and stage III disease only, the 3tier system was a significant predictor of DSS (Fig. $6)(\mathrm{p}<0.02)$, but it did not predict DFS $(\mathrm{p}<0.1986)$. However, a significant separation of the survival curves was evident when the 2-tier system was used, for both DSS (Fig. 7; maximal separation of curves $30 \%$ units $)(\mathrm{p}<0.009)$ and DFS $(\mathrm{p}<0.0106)($ data not shown). These results suggest the potential of DNA cytometry in finding the patients at high risk for clinically progressive disease.

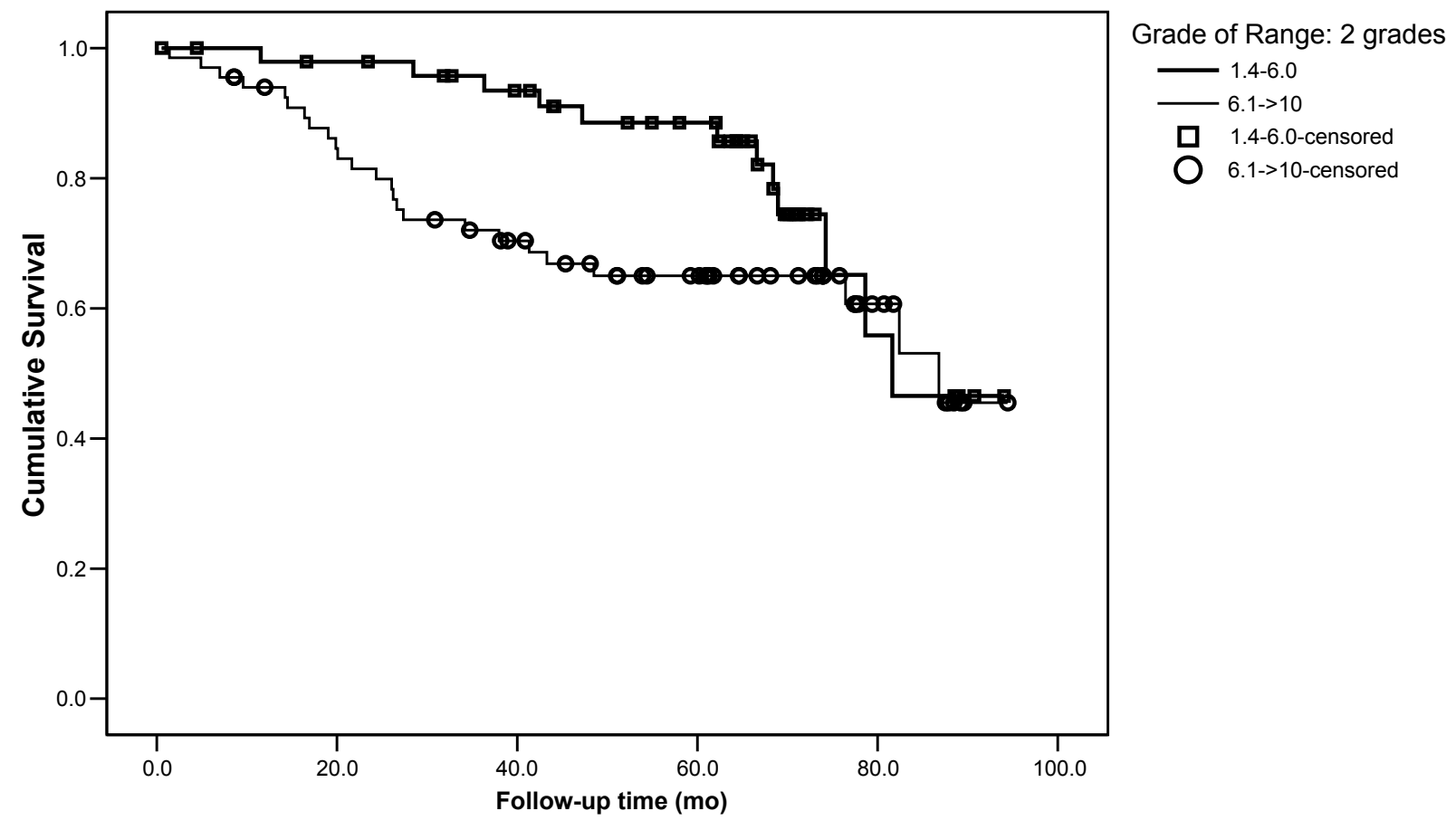

Fig. 5. Histogram width (2-tier grading) in predicting disease-free survival (DFS) in stage II, stage III and stage IV patients. The survival curves represent the 2 grades of the histogram width: Narrow range histograms (1.4-6.0 units, 60 pts) and wide range histograms (6.0-<10.0 units, 67 pts) (Kaplan-Meier analysis; log-rank $p=0.108)$. 


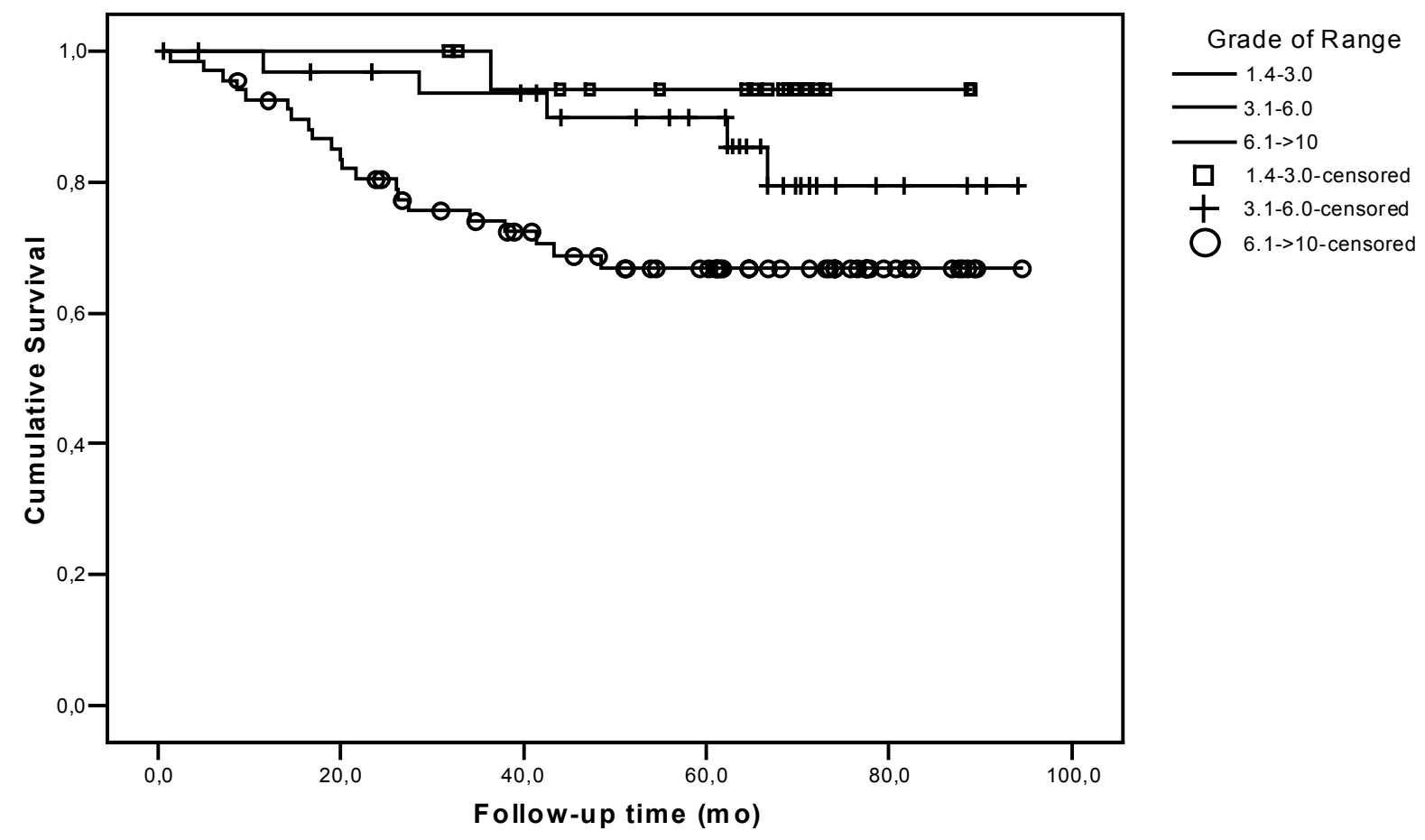

Fig. 6. Histogram width (3-tier grading) in predicting disease-specific survival (DSS) in stage II and stage III patients. The survival curves represent the 3 grades of the histogram width: Narrow range histograms (1.4-3.0 units, 13 pts); Intermediate range histograms (3.1-6.0 units, 29 pts); wide range histograms (>6.0 units, 58 $p t s)$, and deviate from each other significantly (Kaplan-Meier analysis; log-rank $p=0.028$ ).

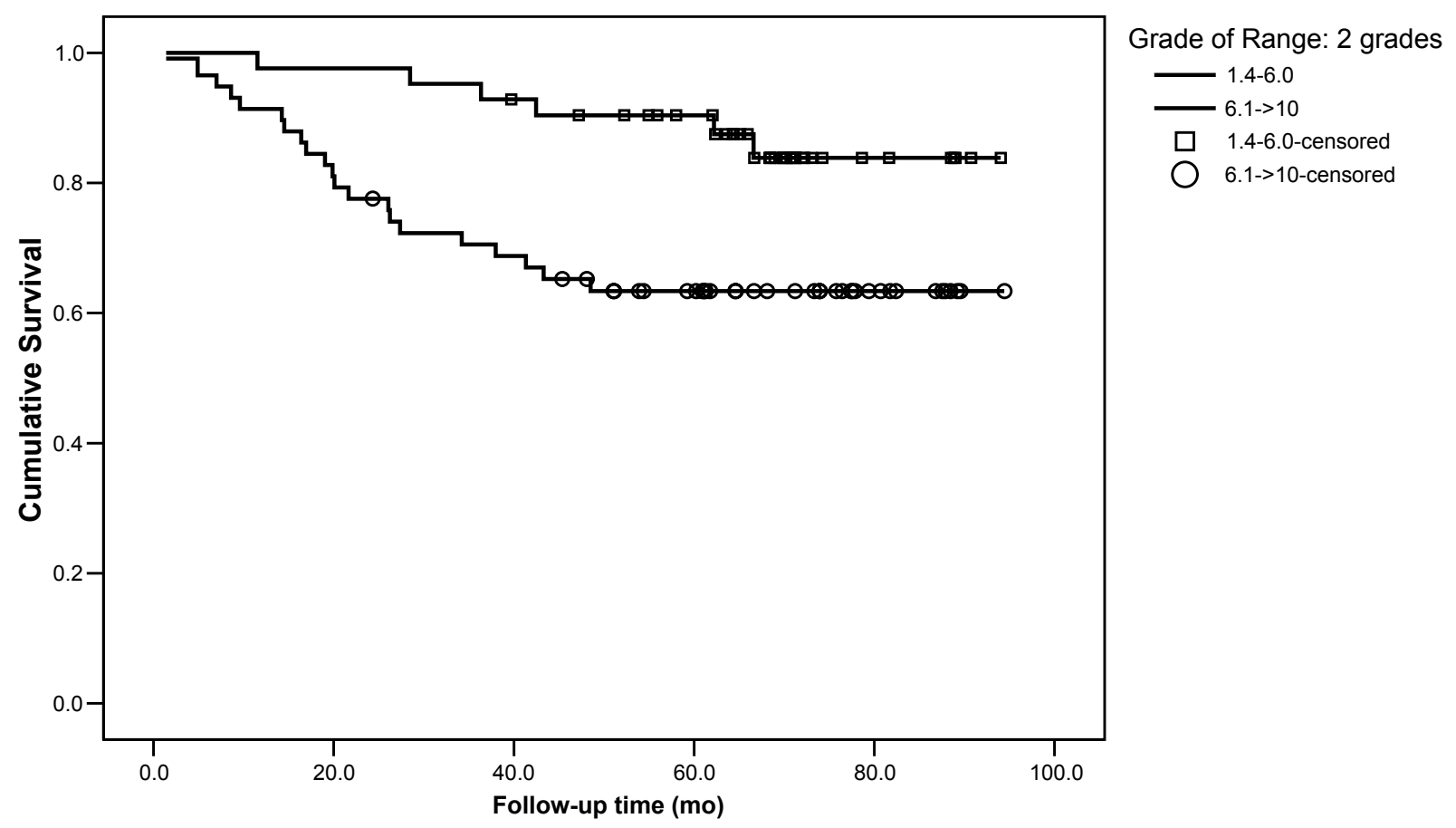

Fig. 7. Histogram width (2-tier grading) in predicting disease-specific survival (DSS) in stage II and stage III patients. The survival curves represent the 2 grades of the histogram width: Narrow range histograms (1.4-6.0 units, 42 pts) and wide range histograms (6.0-<10.0 units, 58 pts), and deviate from each other significantly (Kaplan-Meier analysis; log-rank $p<0.009$ ). 


\section{DISCUSSION}

Genomic instability is observed in the majority of human tumors, and dysregulation of the mitotic spindle checkpoint is thought to be one of the mechanisms that facilitate the development of aneuploidy in the tumor cells (Jaffrey et al., 2000). However, the much of the mechanisms behind genetic instability and aneuploidy still remains unexplored (Giaretti et al., 2004).

CRC develops through a multi-step process with an accumulation of multiple genetic alterations. By estimating chromosomal instability (CIN) and/or microsatellite instability (MSI), one gets an overall impression of this genomic instability. The CIN phenotype is found in approximately $85 \%$ of the sporadic CRCs and is characterized by aneuploidy and multiple chromosomal rearrangements. The MSI phenotype, on the other hand, is associated with small insertions and deletions mainly in repetitive sequences (microsatellites) and is found in approximately $15 \%$ of the cases, being characterized by diploidy or near diploidy (Westra et al., 2004; Rajagopalan and Lengauer, 2004a).

Aneuploidy is one of the features of cancer cells that distinguish them from normal cells (Rajagopalan and Lengauer, 2004b). Because aneuploidy has been recognized as a cardinal feature of many cancers playing an important part in tumorigenesis, it is also a potential therapeutic target, currently under investigation.

There are controversies regarding the role of DNA content as a prognostic factor in human cancer. At least part of this controversy can be explained by the inconsistencies in recording the DNA contents. This was also shown by our previous study on stage II and stage III cases (Buhmeida et al., 2005), where only some of the analytical approaches used resulted in prognostically valuable information. Accordingly, using the $\mathrm{ABCDE}$ system, the histogram patterns were similar in patients who were alive and those who died of disease, which means that this approach of analysis did not able to extract useful information regarding the survival and the same was true with the modal peak evaluations (for both sets). However, when the histogram width (range evaluation) was used to predict the outcome (alive/died), significant differences were disclosed. Indeed, $77.8 \%$ of the patients who died of the disease showed the widest histogram width, as compared to $50.7 \%$ of those who were still alive. In practical terms, the narrow range was an infrequent pattern in patients who died of the disease, and a narrow range usually predicted a favourable outcome (i.e., survival).

We feel that prospective studies on the value of image DNA cytometry in CRC are urgently needed. The true value of DNA measurements can only be evaluated in a study setting where staging is done carefully and in a consistent way, which is rarely possible in retrospective studies. Thus, in the evaluation of DNA cytometry in CRC, it is not only the DNA cytometry itself which should be standardized but also the staging of the disease should be done carefully and in a consistent and comprehensive manner.

There is little doubt that genomic instability is reflected as the wide range histograms, and most of these wide range histograms show DNA aneuploidy. In line with this, the ABCDE system, which does not concentrate on range, did not predict either DFS or DSS in Kaplan-Meier analysis. In contrast, the, modal peak evaluation showed that DFS was shortest for the patients with the modal peak at gate $(6.0-7.7 \mathrm{c})$ (28.9 months) and for those with modal peak at gate (4.3-6.0c) (37.8 months). The value of high DNA values was even more convincingly demonstrated by the analysis of the histogram width (range evaluation). The first set (gates 1-6) was a significant predictor of DFS in Kaplan-Meier analysis, showing that the disease recurrence was closely associated with the histograms of the widest range $1.8 \mathrm{c}->10 \mathrm{c}$. Even more importantly, the first set proved to be a significant predictor of DSS as well.

When these individual histogram gates were lumped together to form a 3-tier system or a 2-tier system of grading, indeed, survival was markedly better (77.8-90.0\%) among the patients with the narrow range histograms. It declined rapidly among the patients with intermediate range histograms and dramatically dropped among the patients with the wide range histograms. The histogram width appeared as a significant predictor of DSS. When applied to predict DFS, the result was not significant, but useful information could still be obtained by viewing the survival curves. It is evident that recurrence rate is markedly higher among the patients with wide range histograms. Thus, by the end of 24-month follow-up, over $20 \%$ more of the patients with wide range histograms have developed a relapse, as compared to those with narrow range histograms.

It is important to notice that associations of DNA histogram features were often different with DFS and DSS. One of the reasons is that these two measures evaluate different things. It is also obvious that great 
difficulties are often encountered in accurately defining DSS, especially if autopsies are not performed for all patients. The classification of patients into the DFS categories (disease present/absent) is probably more consistent in practice.

These data implicate that patients with wide histograms should require closer monitoring at regular intervals. The fact that significant observations are made with some (but not all) of the interpretation methods of the histograms only is another example of the complexity of the DNA patterns. It is obvious that histogram interpretation should be as comprehensive as possible so as to reveal all survival-associated characteristics. In this respect, it is encouraging that the method which gave the best prediction of survival also had high rate of reproducibility in our analysis. One also has to stress that these results are based on analysis of isolated nuclei. DNA cytometry measurements directly from the histological sections are practically impossible, due to the overlaps and cutting of the nuclei.

\section{ACKNOWLEDGEMENTS}

This study has been supported in part by the Special Government Funding (EVO) to Turku University Central Hospital. We would like to thank Mrs. Sinikka Kollanus for her expert assistance in the laboratory work.

\section{REFERENCES}

Aaltonen L (1996) The multistep nature of colon carcinogenesis. Cytokines Cell Mol Ther 2:111-4.

Bazan V, Migliavacca M, Zanna I, Tubiolo C, Corsale S, Calo V, et al. (2002). DNA ploidy and S-phase fraction, but not $\mathrm{p} 53$ or NM23-H1 expression, predict outcome in colorectal cancer patients. Result of a 5-year prospective study. Cancer Res Clin Oncol 128(12): 650-8.

Bendardaf R, Lamlum H, Pyrhonen S (2004a). Prognostic and predictive molecular markers in colorectal carcinoma. Anticancer Res 24(4):2519-30.

Bendardaf R, Lamlum H, Ristamaki R, Algars A, Collan Y, Pyrhonen S (2004b). Response to chemotherapy (irinotecan plus 5-fluorouracil) in colorectal carcinoma can be predicted by tumour DNA content. Oncology 66(1):46-52.

Bohnhoff GL (1979). Setting up proper illumination on your microscope. Am J Med Technol 45(7):650-1.

Cohen C (1996). Image cytometric analysis in pathology. Hum Pathol 27(5):482-93.

Fearon ER, Vogelstein B (1990). A genetic model for colorectal tumorigenesis. Cell 61(5):759-67.
Galizia G, Orditura M, Romano C, Lieto E, Castellano P, Pelosio L, et al. (2002). Prognostic significance of circulating IL-10 and IL-6 serum levels in colon cancer patients undergoing surgery. Clin Immunol 102(2): 169-78.

Gaub J, Auer GU, Zetterberg A (1975). Quantitive cytological aspects of a combined Feulgen naphthol yellow S staining procedure for the simultaneous determinatins of nuclear and cytoplasmic protein and DNA in mammalian cells. Exp Cell Res 92: 323-32.

Geido E, Sciutto A, Rubagotti A, Oliani C, Monaco R, Risio M, et al. (2002). Combined DNA flow cytometry and sorting with k-ras2 mutation spectrum analysis and the prognosis of human sporadic colorectal cancer. Cytometry 50(4):216-24.

Giaretti W, Molinu S, Ceccarelli J, Prevosto C (2004). Chromosomal instability, aneuploidy, and gene mutations in human sporadic colorectal adenomas. Cell Oncol 26(5-6):301-5.

Giaretti W, Venesio T, Sciutto A, Prevosto C, Geido E, Risio M. (2003). Near-diploid and near-triploid human sporadic colorectal adenocarcinomas differ for KRAS2 and TP53 mutational status. Genes Chromosomes Cancer 37(2):207-13.

Hawkins NJ, Tomlinson I, Meagher A, Ward RL. (2001). Microsatellite-stable diploid carcinoma: a biologically distinct and aggressive subset of sporadic colorectal cancer. Br J Cancer 84(2):232-6.

Hedley DW, Friedlander ML, Taylor IW (1985). Application of DNA flow cytometry to paraffin-embedded archival material for the study of aneuploidy and its clinical significance. Cytometry 6(4):327-33.

Jaffrey RG, Pritchard SC, Clark C, Murray GI, Cassidy J, Kerr KM, et al. (2000). Genomic instability at the BUB1 locus in colorectal cancer, but not in non-small cell lung cancer. Cancer Res 15;60(16):4349-52.

Lanza G, Gafa R, Santini A, Maestri I, Dubini A, Gilli G, et al. (1998). Prognostic significance of DNA ploidy in patients with stage II and stage III colon carcinoma: a prospective flow cytometric study. Cancer 82(1):49-59.

Millot C, Dufer J (2000). Clinical applications of image cytometry to human tumour analysis. Histol Histopathol 15(4):1185-200.

Petersen SE, Bichel P, Lorentzen M (1979). Flow-cytometric demonstration of tumour-cell subpopulations with different DNA content in human colo-rectal carcinoma. Eur J Cancer 15(4):383-6.

Rajagopalan H, Lengauer C (2004a). CIN-ful cancers. Cancer Chemother Pharmacol 54(1):S65-8.

Rajagopalan H, Lengauer C (2004b). hCDC4 and genetic instability in cancer. Cell Cycle 3(6):693-4.

Salud A, Porcel JM, Raikundalia B, Camplejohn RS, Taub NA (1999). Prognostic significance of DNA ploidy, S-phase fraction, and P-glycoprotein expression in colorectal cancer. J Surg Oncol 72(3):167-74. 
Steinbeck RG (1998). Chromosome division figures reveal genomic instability in tumorigenesis of human colon mucosa. Br J Cancer 77(7):1027-33.

Scott NA, Wieand HS, Moertel CG, Cha SS, Beart RW, Lieber MM (1987). Colorectal cancer. Dukes' stage, tumor site, preoperative plasma CEA level, and patient prognosis related to tumor DNA ploidy pattern. Arch Surg 122(12):1375-9.

Silvestrini R (2000). Relevance of DNA-ploidy as a prognostic instrument for solid tumors. Ann Oncol 11(3):259-61.

Sugai T, Takahashi H, Habano W, Nakamura S, Sato K, Orii S, et al. (2003). Analysis of genetic alterations, classified according to their DNA ploidy pattern, in the progression of colorectal adenomas and early colorectal carcinomas. J Pathol 2:168-76.

Tomlinson I, Ilyas M, Novelli M (1997). Molecular genetics of colon cancer. Cancer Metastasis Rev 16:67-79.

Tonouchi H, Matsumoto K, Kinoshita T, Itoh H, Suzuki H. (1998). Prognostic value of DNA ploidy patterns of colorectal adenocarcinoma: univariate and multivariate analysis. Dig Surg 15(6):687-92.

Tribukait B, Hammarberg C, Rubio C (1983). Ploidy and proliferation patterns in colo-rectal adenocarcinomas related to Dukes' classification and to histopathological differentiation. A flow-cytometric DNA study. Acta Pathol Microbiol Immunol Scand 91(2):89-95.

Westra JL, Plukker JT, Buys CH, Hofstra RM. (2004). Genetic alterations in locally advanced stage II/III colon cancer: a search for prognostic markers. Clin Colorectal Cancer 4(4):252-9.

Witzig TE, Loprinzi CL, Gonchoroff NJ, Reiman HM, Cha SS, Wieand HS, et al. (1991). DNA ploidy and cell kinetic measurements as predictors of recurrence and survival in stages B2 and C colorectal adenocarcinoma. Cancer 15;68(4):879-88.

Zarbo RJ, Nakhleh RE, Brown RD, Kubus JJ, Ma CK, Mackowiak P. (1997). Prognostic significance of DNA ploidy and proliferation in 309 colorectal carcinomas as determined by two-color multiparametric DNA flow cytometry. Cancer 79(11):2073-86. 